Int. J. Curr. Res. Med. Sci. (2017). 3(5): 84-99

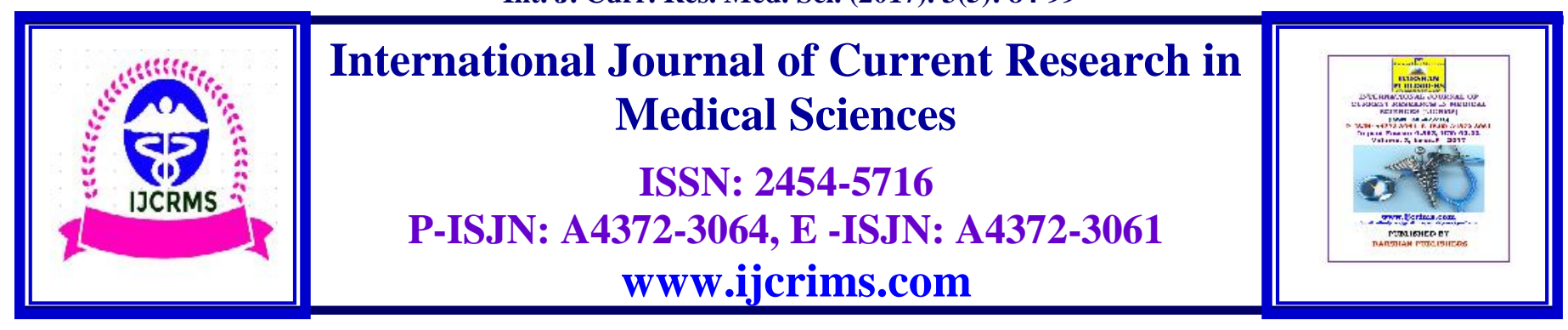

\title{
Investigation and evaluation of acute and sub-acute dermal toxicity studies of ethanolic leaves extract of Melastoma malabathricum in Sprague Dawley rats
}

\author{
Ali Khairullah Zahi ${ }^{1}$, Hazilawati Hamzah ${ }^{1}$, Mohd Rosly Shaari ${ }^{2}$, \\ Riyanto Teguh Widodo ${ }^{3}$, Lucy Johnny ${ }^{4}$, Noordin M.M ${ }^{1}$, and \\ Shanmugavelu Sithambaram ${ }^{2}$ \\ ${ }^{1}$ Department of Pathology and Microbiology, Faculty of Veterinary Medicine, University Putra Malaysia, \\ 43400 UPM Serdang, Selangor, Malaysia. \\ ${ }^{2}$ Department of Deputy Director, Strategic Livestock Research Centre, Malaysia Agricultural Research and \\ Development Institute, 43400 Serdang, Selangor, Malaysia. \\ ${ }^{3}$ Department of Pharmacy, Faculty of Medicine, University of Malaya, 50603 Kuala Lumpur, Malaysia. \\ ${ }^{4}$ Sunway College KL, 46150 Bandar Sunway, Selangor, Malaysia \\ *Corresponding author: alikhfaghi@gmail.com
}

\begin{abstract}
Melastoma malabathricum is an important plant commonly used in traditional medicine. Until recently, the dermal toxicity profile of $M$. malabathricum remained unknown. The objective of this study is to investigate the in vivo acute and sub-acute dermal toxicity of ethanolic leaves extract of $M$. malabathricum in rats. In acute experiment, a total of 20 female rats were divided into four groups, each group had five rats. While, a total of 30 male rats were divided into five groups, each group consisted of six rats in sub-acute experiment. Single doses of the extract at 2000 and 5000 $\mathrm{mg} / \mathrm{kg}$ of body weight failed to produce treatment-related signs of toxicity or mortality during the 14-day observation period. In a repeated dose 28-day study, 500, 1000 and $2000 \mathrm{mg} / \mathrm{kg}$ of body weight/day applications of leaf extract lead to no significant change $(\mathrm{p}>0.05)$ in bodyweight or haematological and biochemical parameters compared with the control group. Similarly, gross pathology and histopathology examinations of liver, spleen, kidneys, and skin did not reveal any morphological alteration. Results indicate that the close application of M. malabathricum leaves extract had no critically dangerous effect on the rats tested. Therefore, the concentrate may be used pharmaceutically.
\end{abstract}

Keywords: Melastoma malabathricum, acute toxicity, sub-acute toxicity, histopathology.

\section{Introduction}

Melastomataceae plants originate in tropic and subtropical regions. There is a total of more than 4000 species worldwide. In just South-East Asia region alone, the genus Melastoma comprises 22 species, 2 subspecies, and 3 varieties (Rajenderan, 2010). Malaysia, particularly, with a tropical 
climate, is home to at least 12 species, many of which are used by natives in folk medicine. One of the plants within the Melastomataceae family with a status in Malay folklore belief is Melastoma malabathricum L., which has been known to comprise two subspecies, namely M. malabathricum L. ssp. malabathricum and M. malabathricum Linn ssp. normale (Meyer, 2001). The characteristics of $M$. malabathricum include its average height of (0.5-1 m) high but may occasionally grow up to $5 \mathrm{~m}$ long (Zakaria and Mohd, 1994). Melastoma is Greek for "black mouth", a name appreciated by generations of children who have eaten the berries. M. malabathricum has evergreens and flowers throughout the year (Koay, 2008).

In addition, M. malabathricum leaves, flowers, seeds, and roots are used in traditional medicine to treat a variety of ailments as skin diseases, venereal diseases and diabetes as well as having a workable and neat anti-inflammatory and antimicrobial effects (Grosvenor et al., 1995; Sunilson et al., 2008). Hazardous chemicals may lead to adverse health effects in humans. Plants used in alternative medicine may contain toxic compounds. Toxicity refers to chemicals within the plant being poisonous to cells. Typically, exposure to toxic compounds leads to signs of acute toxic effects. Proper evaluation of these effects protects the public's health. One form of toxicological assessment is animal testing of potential remedies. OECD guidelines allow testing on animals such as rabbits, monkeys, guinea pigs, and dogs. In practical terms, evaluations must consider acute, sub-acute, subchronic, chronic, carcinogenic, and reproductive effects (Asante-Duah, 2002).

This study examines the use of this extract in topical applications. Both acute and sub-acute dermal toxicity studies are performed. Acute toxicity is measured using a single large dose to determine both immediate toxic effects and LD50. The sub-acute toxicity test uses sub-lethal doses over 15 to 30 days to determine the no observed adverse effect level or NOAEL (Lipnick et al., 1995). This study examines the toxic effects of ethanolic leaf extract of $M$. malabathricum in Sprague Dawley rats at dosages of 2000 and 5000 $\mathrm{mg} / \mathrm{kg}$ body weight for a period of 14 days for acute toxicity study per OECD 402 guidelines, and at dosages of 2000, 1000 and $500 \mathrm{mg} / \mathrm{kg}$ body weight for a period of 28 days for sub-acute toxicity study per OECD 410 guidelines.

\section{Methods}

\section{Experimental animals:}

Male and female Sprague Dawley rats with a body weight ranging from 200 to $250 \mathrm{~g}$ were purchased from a local supplier at eight weeks old. Upon arrival, the rats were weighed and assigned randomly in polypropylene plastic cages, where one rat was placed in each cage with wood chips for bedding and housed in an animal room with controlled conditions involving these parameters; temperature $\left(22 \pm 2^{\circ} \mathrm{C}\right)$, humidity $(55 \pm 10 \%)$ and lighting (12 hours light/dark) in the animal house at the Malaysian Agricultural Research and Development Institute (MARDI), Serdang, Selangor.

\section{Preparation of Melastoma malabathricum (Senduduk) leaf extracts}

Fresh, whole plants were obtained from Jerantut, Pahang, Malaysia. After separating the leaves, they were dried at temperature between $25^{\circ} \mathrm{C}$ to $30^{\circ} \mathrm{C}$ for three to five days. The dried leaves were then ground into powder via a grinder and placed in a refrigerator at $4^{\circ} \mathrm{C}$. After that, the powdered leaves were macerated in a flask with $97 \%$ ethanol. The mixture underwent a water bath at temperature between $55^{\circ} \mathrm{C}$ to $60^{\circ} \mathrm{C}$ for one day in order for the chemicals in the leaves to become fully dissolved in the ethanol solution. The ethanol was isolated from the mixture via filtration followed by evaporation using a rotor paper evaporator. The remaining elements were then dried via a freeze dryer for one day. The diluted solutions were stored in a refrigerator for later use. The leaf extract was mixed with white soft paraffin (10\%).

\section{Skin preparation for dermal toxicity studies}

The skin at the dorsal thoracic area of the rats was clipped under general ketamine $(50 \mathrm{mg} / \mathrm{kg})$ and xylazine $(5 \mathrm{mg} / \mathrm{kg})$ anaesthesia with an electric clipper. This was followed by manual shaving 
using a razor blade. Based on OECD guidelines 402 and 410, not less than $10 \%$ of the body surface area was cleared for the application of the test substance. The extract was applied to dorsum area.

\section{Experimental design}

To determine acute and sub-acute dermal toxicity, the toxic effects of the ethanolic leaf extract of M. malabathricum in Sprague Dawley rats were ascertained at dosages of 2000 and $5000 \mathrm{mg} / \mathrm{kg}$ body weight for a period of 14 days (acute dermal toxicity), and at dosages of 2000, 1000 and 500 $\mathrm{mg} / \mathrm{kg}$ body weight for a period of 28 days (subacute dermal toxicity) per OECD guidelines 402 and 410, respectively. Different doses were used to ensure that this extract is not toxic and safe in different concentrations. Base on OECD guidelines, female rats are more sensitive to toxic substances than males in acute toxicity studies. The application of extract in acute toxicity study is once but in sub-acute dermal toxicity is daily, thus female rats used in acute dermal toxicity and male used in sub-acute dermal toxicity. In acute dermal toxicity, a total number of 20 eight-week old female Sprague Dawley rats were divided into four groups $(n=5)$ namely Groups $1,2,3$ and 4. Duration of this study was 14 days. Each group was applied with the extract once at day 1 and sacrificed at day 14 of the experimental period.

On the other hand, in sub-acute dermal toxicity a total of 30 eight-week old male Sprague Dawley rats were divided into 5 groups $(n=6)$ namely Groups 1, 2, 3, 4 and 5. This study ran for 28 days and each group underwent extract application once daily for 28 days, and all were sacrificed at day 28 of the experimental period.

\section{Necropsy}

All rats were humanely scarified by complete exsanguinations under general anaesthesia with a mixture of $75 \mathrm{mg} / \mathrm{kg}$ Ketamine and $10 \mathrm{mg} / \mathrm{kg}$ Xylazine.

\section{Gross pathology}

Complete gross examination was conducted to detect any gross changes especially skin necrosis.
The liver and kidneys were blotted dry and weighed immediately after necropsy.

\section{Histopathology}

Skin, liver, and kidneys samples were collected and fixed in $10 \%$ formalin for 48 hours. Samples were sliced to $0.5 \mathrm{~cm}$ thickness and placed in plastic cassettes for dehydration using an automated processor (Leica ASP300, Germany). After this step, they were embedded in paraffin (Leica EG1160, Germany). The tissue samples were then trimmed and sectioned at $4 \mathrm{~m}$ thicknesses (Leica RM2155, Germany). Mounting was carried out on glass slides with a hot plate (Leica HI1220, Germany). Deparaffinization was then carried out via xylene exchange, after which rehydration using different ethanol dilutions (100\%, 90\% and $70 \%$ ) was then performed for 2 minutes each, respectively. The tissue sections were then further rinsed in tap water and stained with Harris's haematoxylin and eosin (H\&E) stain. A light microscope at 40x, 100x, 200x, 400x and 1000x magnification levels was used for observation.

\section{Blood biochemistry}

The blood samples were centrifuged (HettichzentEBA20, Germany) at $5000 \mathrm{rpm}$ for 5 minutes. The serum was then placed into micro centrifuge tubes and kept at $-20^{\circ} \mathrm{C}$. Serum samples were analysed using an automatic biochemistry analyser (TRX 7070, Biorex, Germany). Levels of aspartate transaminase (AST), lactate dehydrogenase (LDH), alanine transaminase (ALT), total protein, albumin, creatinine, uric acid, urea, and bilirubin were measured. Globulin levels were determined via the formula (total protein - albumin).

\section{Haematology}

Blood samples were taken from the posterior vena cava of each rat. Samples were then placed into ethylene diamine tetra acetic acid (EDTA) vacuumed blood collection tubes and gently mixed with EDTA-anticoagulant material for both automatic and manual haematology analyses. 


\section{Automatic haematology}

Blood samples were analysed via an automatic haematology analyser (Cell Dyn, 3700, Abbot, USA) for the total number of white blood cell (WBC), red blood cell (RBC) and haemoglobin concentration.

\section{Manual haematology}

EDTA blood was placed via capillary action into a capillary microhematocrit tube to about a level of about three-fourths of its length. The dry end of microhematocrit tubes was sealed by melting, then placed in a micro-centrifuge machine (HettichHaematokrit 210, Germany). It was then centrifuged at $10,000 \mathrm{rpm}$ for 5 minutes to separate the RBCs from plasma. The plasma rose to the top of the microhematocrit tube while the RBCs went to the bottom. The centrifuged microhematocrit tubes were used for determination of packed cell volume (PCV), icteric index and plasma protein concentration. For the PCV, the microhematocrit tube was placed in holder slots of the microhematocrit tube reader (Hawksley), where the base of red blood cell was intersected with base line of reader and the top of plasma was intersected with the top line of the reader by moving the holder left or right, before the middle line of the reader was adjusted to intersect with the top of the RBCs and the measuring ruler. PCV results were obtained from the middle line and the measuring ruler point (e.g.: $24 \%$ is equal to $0.24 \mathrm{~L}$ ). Icteric index results were obtained by comparing plasma colour in the microhaematocrit tube with the icteric index standard board colour degree. While the blood plasma protein concentration was obtained by dropping the plasma on the refractometer glass (Atago T2-NE, Japan) to obtain the result from the measuring ruler and the plasma unit was read in protein in gram divided by plasma in litre (e.g.: $6.2 \%$ is equal to $62 \mathrm{~g} / \mathrm{L}$ ).

\section{Peripheral blood smear}

A peripheral blood smear was performed. First, a small blood drop was placed on a glass slide. Then, a drop smeared using a cover slip on the slide surface. This was left at room temperature for 20 minutes to dry. The blood smear was stained via Wright's stain method. The blood smear slide underwent examination with a light microscope at 100x, 200x, 400x, and 1000x magnifications.

A manual WBC differential count was carried out for one hundred WBCs. The values for each WBC type were converted to a percentage. Then, these values were multiplied by the automated total WBC count to obtain absolute WBC differential counts $(\times 109 / \mathrm{L})$.

\section{Statistical analysis}

The data obtained were statistically analysed by using Statistical Package for Social Science (SPSS) software version 20. The values were expressed as mean \pm standard deviation (SD) for different parameters. Repeated measurements of analysis of variance (ANOVA) tests were done to compare the differences of data between and within the groups. A Duncan test was conducted for post hoc analysis. The level of statistical significance was set at $\mathrm{P}<0.05$.

\section{Results}

\section{Acute Toxicity Study}

\section{General sign and behaviour of the rats}

M. malabathricum ethanolic extracts' effects on appearance and general behavioural patterns are shown in Table 1 and Table 2, respectively. No toxic signs or mortality were observed in any animals, which survived up to 14 days after applying of the extracts once on the first day at single dose level of 5000 and $2000 \mathrm{mg} / \mathrm{kg}$ body weight. Behavioural patterns were observed both for the first 6 hours and 14 hours after extract application. The animals in both vehicle-treated and extract-treated groups were normal and did not display significant changes in behaviour, skin effects, breathing, impairment in food intake and water consumption, postural abnormalities and hair loss. Treated groups showed a rapid heartbeat for the first 6 hours, but soon normalized. This is likely due to stresses endured during handling. 
Table 1: Mortality rate of rats after applied with topical ethanolic extracts of $M$. malabathricum at $5000 \mathrm{mg} / \mathrm{kg}$ and $2000 \mathrm{mg} / \mathrm{kg}$, once, for the acute toxicity study

\begin{tabular}{|c|c|}
\hline Group & Mortality rate*(\%) \\
\hline G1 & 0 \\
\hline G2 & 0 \\
\hline G3 & 0 \\
\hline G4 & 0 \\
\hline
\end{tabular}

* Mortality rate is number of dead rats divided by total number of rats per group.G1: No treatment, G2: Paraffin, G3: M. malabathricum 5000 mg/kg, G4: M. malabathricum 2000 mg/kg

Table 2: Behavioural patterns and general appearance of rats in all groups

\begin{tabular}{|c|c|c|c|c|}
\hline Abnormal sign & $\begin{array}{c}\text { Control } \\
\text { group: } \\
\text { G1 }(6 \mathrm{~h})\end{array}$ & $\begin{array}{c}\text { Control group: } \\
\text { G1 }(14 \mathrm{~h})\end{array}$ & $\begin{array}{c}\text { Treatment } \\
\text { groups: } \\
\text { G2-G4 }(6 \mathrm{~h})\end{array}$ & $\begin{array}{c}\text { Treatment } \\
\text { groups: } \\
\text { G2-G4 }(14 \mathrm{~h})\end{array}$ \\
\hline Skin and fur & Normal & Normal & Normal & Normal \\
\hline Eyes & Normal & Normal & Normal & Normal \\
\hline Mucous membrane & Normal & Normal & Normal & Normal \\
\hline Behavioural patterns & Normal & Normal & Tachycardia & Normal \\
\hline Salivation & Normal & Normal & Normal & Normal \\
\hline Lethargy & Normal & Normal & Normal & Normal \\
\hline Sleep & Normal & Normal & Normal & Normal \\
\hline Diarrhea & Normal & Normal & Normal & Normal \\
\hline Coma & NO & NO & NO & NO \\
\hline Tremors & NO & NO & NO & NO \\
\hline
\end{tabular}

G1: No treatment, G2: Paraffin, G3: M. malabathricum 5000 mg/kg, G4: M. malabathricum $2000 \mathrm{mg} / \mathrm{kg}$. NO: Not observed

\section{Organ and body weight}

Body weights and weights of liver and kidneys are shown in Figure 1 and Table 3. No significant changes in the body weight were found. All rats had shown a normal increment in the body weight which was not significantly $(\mathrm{p}>0.05)$ different between both control and treated groups. As with body weight, no significant $(\mathrm{p}>0.05)$ differences in the changes of relative organ weights between groups were noted.

Table 3: Organ relative weights of rats in all groups

\begin{tabular}{|c|c|c|}
\hline Group & Liver $(\mathrm{g})$ & Kidneys $(\mathrm{g})$ \\
\hline G1 & $0.024 \pm 0.005$ & $0.005 \pm 0.0005$ \\
\hline G2 & $0.020 \pm 0.007$ & $0.006 \pm 0.0004$ \\
\hline G3 & $0.026 \pm 0.005$ & $0.005 \pm 0.0005$ \\
\hline G4 & $0.024 \pm 0.008$ & $0.005 \pm 0.0008$ \\
\hline
\end{tabular}

Values are expressed as mean $\pm \mathrm{SD}$ ( $n=5$ for each group). Relative organ weight was calculated by organ weight/body weight $\times 100 \%$. G1: No treatment, G2: Paraffin, G3: M. malabathricum $5000 \mathrm{mg} / \mathrm{kg}$, G4: M. malabathricum $2000 \mathrm{mg} / \mathrm{kg}$ 
Int. J. Curr. Res. Med. Sci. (2017). 3(5): 84-99

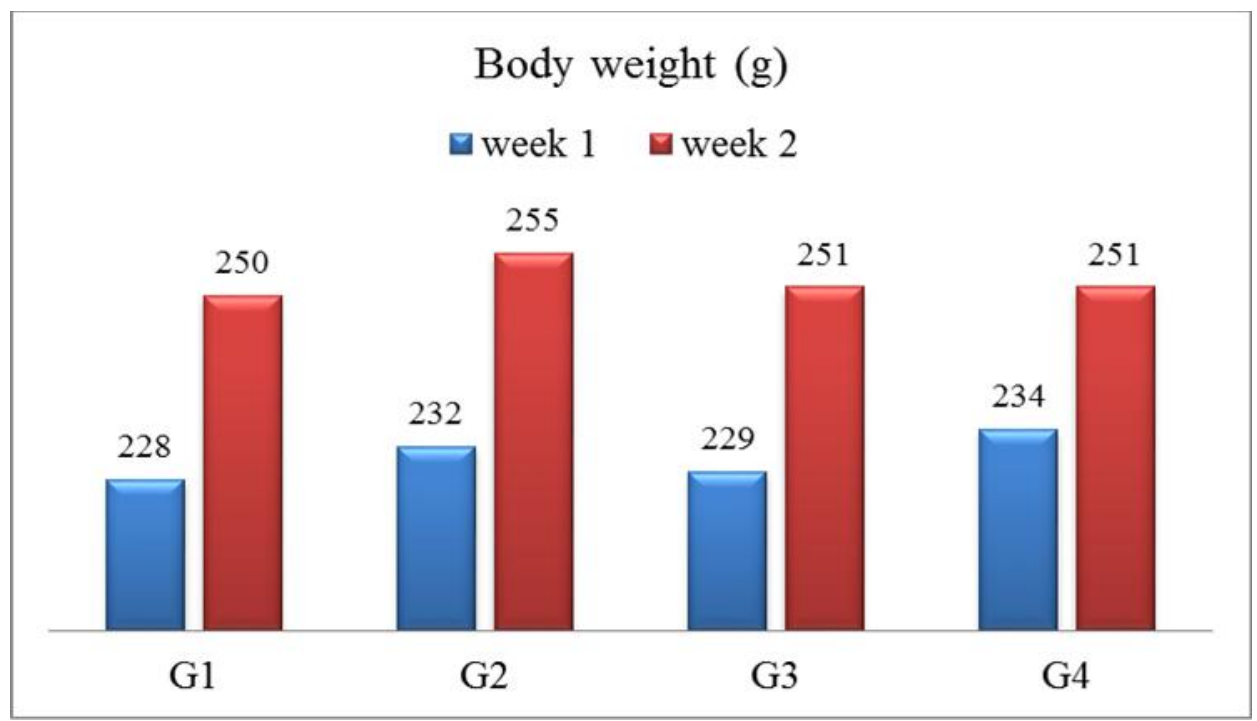

Fig. 1: Mean body weight (g) of rats in all groups. Data collected were recorded and presented as mean \pm standard error of mean. G1: No treatment; G2: Paraffin; G3: M. malabathricum $2000 \mathrm{mg} / \mathrm{kg}$; G4: M. malabathricum $5000 \mathrm{mg} / \mathrm{kg}$. None of the numbers were seriously variant at $\mathrm{p}>0.05$

\section{Haematology evaluation}

All erythron parameters were normal in all rats as shown in Table 4. Results were also normal for the leukon parameters, which are depicted in Table 5 .

Table 4: Erythron parameters and plasma protein concentration of rats in all groups

\begin{tabular}{|c|c|c|c|c|c|}
\hline Parameter & Unit & $\begin{array}{l}\text { G1(Mean } \\
\pm \text { SD })\end{array}$ & $\begin{array}{l}\text { G2(Mean } \\
\pm \text { SD) }\end{array}$ & $\begin{array}{l}\text { G3(Mean } \\
\pm S D)\end{array}$ & $\begin{array}{l}\text { G4(Mean } \\
\pm S D)\end{array}$ \\
\hline $\mathrm{RBC}$ & $\mathrm{x} 1012 / \mathrm{L}$ & $8.25 \pm 0.28$ & $8.56 \pm 0.61$ & $8.66 \pm 0.60$ & $8.43 \pm 0.83$ \\
\hline $\mathrm{Hb}$ & $\mathrm{g} / \mathrm{L}$ & $171 \pm 7.91$ & $166 \pm 7.79$ & $178 \pm 8.90$ & $169 \pm 16.69$ \\
\hline $\mathrm{PCV}$ & $\mathrm{L} / \mathrm{L}$ & $0.45 \pm 0.00$ & $0.45 \pm 0.01$ & $0.45 \pm 0.00$ & $0.45 \pm 0.02$ \\
\hline $\mathrm{MCV}$ & $\mathrm{fl}$ & $58.2 \pm 0.83$ & $57.8 \pm 1.30$ & $56.6 \pm 1.51$ & $56.6 \pm 1.51$ \\
\hline $\mathrm{PP}$ & $\mathrm{g} / \mathrm{L}$ & $75.4 \pm 2.07$ & $77.8 \pm 1.92$ & $77.0 \pm 3.16$ & $75.4 \pm 1.14$ \\
\hline
\end{tabular}

Values are expressed as mean $\pm \mathrm{SD}$ ( $n=5$ for each group). G1: No treatment; G2: Paraffin; G3:M. malabathricum $5000 \mathrm{mg} / \mathrm{kg}$; G4: M. malabathricum $2000 \mathrm{mg} / \mathrm{kg}$

Table 5: Leukon and thrombon parameters of rats in all groups

\begin{tabular}{|c|c|c|c|c|c|}
\hline Parameter & Unit & $\begin{array}{l}\text { G1(Mean } \\
\pm \text { SD) }\end{array}$ & $\begin{array}{l}\text { G2(Mean } \\
\pm \text { SD) }\end{array}$ & $\begin{array}{l}\text { G3(Mean } \\
\pm S D)\end{array}$ & $\begin{array}{l}\text { G4(Mean } \\
\pm S D)\end{array}$ \\
\hline WBC & $10^{9} / \mathrm{L}$ & $7.82 \pm 0.87$ & $7.68 \pm 0.93$ & $7.80 \pm 1.05$ & $7.52 \pm 1.02$ \\
\hline Neutrophils & $10^{9} / \mathrm{L}$ & $1.45 \pm 1.14$ & $1.47 \pm 0.83$ & $1.43 \pm 0.89$ & $1.36 \pm 0.83$ \\
\hline Lymphocytes & $10^{9} / \mathrm{L}$ & $5.66 \pm 1.14$ & $5.66 \pm 1.64$ & $5.89 \pm 1.67$ & $5.56 \pm 1.22$ \\
\hline Monocytes & $10^{9} / \mathrm{L}$ & $0.31 \pm 1.00$ & $0.32 \pm 1.30$ & $0.29 \pm 0.83$ & $0.36 \pm 0.83$ \\
\hline Esoinophils & $10^{9} / \mathrm{L}$ & $0.21 \pm 0.83$ & $0.12 \pm 0.89$ & $0.09 \pm 0.83$ & $0.12 \pm 0.54$ \\
\hline Basophils & $10^{9} / \mathrm{L}$ & $0.07 \pm 0.70$ & $0.09 \pm 0.83$ & $0.07 \pm 0.70$ & $0.10 \pm 1.14$ \\
\hline Platelets & $10^{9} / \mathrm{L}$ & $869 \pm 40.70$ & $913 \pm 28.10$ & $890 \pm 39.20$ & $888 \pm 33.60$ \\
\hline
\end{tabular}

Values are expressed as mean $\pm \mathrm{SD}$ ( $n=5$ for each group). G1: No treatment; G2: Paraffin; G3:M. malabathricum $5000 \mathrm{mg} / \mathrm{kg}$; G4: M. malabathricum $2000 \mathrm{mg} / \mathrm{kg}$ 


\section{Blood biochemistry}

The serum biochemical parameters of liver and muscle enzymes are shown below. Table 6 depicts the kidney parameters, while Table 7 shows protein concentration and Table 8 contains information for the control female rats and M. malabathricum extract groups. No significant ( $\mathrm{p}>0.05$ ) changes were observed for any biochemical parameter.

Table 6: Serum biochemical parameters of liver and muscle enzymes of rats in all groups

\begin{tabular}{|c|l|l|l|l|l|}
\hline Parameter & Unit & $\begin{array}{l}\text { G1(Mean } \\
\pm \text { SD) }\end{array}$ & $\begin{array}{l}\text { G2(Mean } \\
\pm \text { SD) }\end{array}$ & $\begin{array}{l}\text { G3(Mean } \\
\pm \text { SD) }\end{array}$ & $\begin{array}{l}\text { G4(Mean } \\
\pm \text { SD) }\end{array}$ \\
\hline ALT & (U/L) & $41.2 \pm 1.30$ & $43.4 \pm 1.14$ & $41.6 \pm 1.14$ & $43.4 \pm 2.07$ \\
\hline ALP & (U/L) & $74.0 \pm 2.73$ & $73.2 \pm 2.58$ & $74.2 \pm 1.64$ & $72.0 \pm 2.34$ \\
\hline AST & (U/L) & $134.6 \pm 2.96$ & $139.0 \pm 2.23$ & $139.2 \pm 2.38$ & $138.2 \pm 2.77$ \\
\hline CK & (U/L) & $172.8 \pm 2.68$ & $174.6 \pm 1.81$ & $174.2 \pm 2.16$ & $173.8 \pm 2.16$ \\
\hline
\end{tabular}

Values are expressed as mean $\pm \mathrm{SD}$ ( $n=5$ for each group). G1: No treatment; G2: Paraffin;

G3: M. malabathricum 5000 mg/kg; G4: M. malabathricum 2000 mg/kg

Table 7: Serum biochemical parameters of kidney of rats in all groups

\begin{tabular}{|c|c|l|l|l|l|}
\hline Parameter & Unit & $\begin{array}{l}\text { G1(Mean } \\
\pm \text { SD })\end{array}$ & $\begin{array}{l}\text { G2(Mean } \\
\pm \text { SD })\end{array}$ & $\begin{array}{l}\text { G3(Mean } \\
\pm S D)\end{array}$ & $\begin{array}{l}\text { G4(Mean } \\
\pm S D)\end{array}$ \\
\hline Urea & $(\mathrm{mmol} / \mathrm{L})$ & $6.44 \pm 0.25$ & $6.30 \pm 0.30$ & $6.70 \pm 0.15$ & $6.40 \pm 0.27$ \\
\hline Creatinine & $(\mathrm{mol} / \mathrm{L})$ & $36.5 \pm 1.35$ & $37.5 \pm 1.46$ & $37.6 \pm 1.84$ & $36.3 \pm 2.86$ \\
\hline
\end{tabular}

Values are expressed as mean \pm SD ( $n=5$ for each group). G1: No treatment; G2: Paraffin;

G3: M. malabathricum $5000 \mathrm{mg} / \mathrm{kg}$; G4: M. malabathricum 2000 mg/kg

Table 8: Serum biochemical parameters of protein concentration of rats in all groups

\begin{tabular}{|c|c|l|l|l|l|}
\hline Parameter & Unit & $\begin{array}{l}\text { G1(Mean } \\
\pm \text { SD })\end{array}$ & $\begin{array}{l}\text { G2(Mean } \\
\pm \text { SD })\end{array}$ & $\begin{array}{l}\text { G3(Mean } \\
\pm S D)\end{array}$ & $\begin{array}{l}\text { G4(Mean } \\
\pm S D)\end{array}$ \\
\hline TP & $(\mathrm{g} / \mathrm{L})$ & $72.8 \pm 1.92$ & $73.8 \pm 1.92$ & $73.4 \pm 1.14$ & $73.0 \pm 2.54$ \\
\hline Albumin & $(\mathrm{g} / \mathrm{L})$ & $37.7 \pm 0.97$ & $38.1 \pm 0.79$ & $38.1 \pm 0.73$ & $38.5 \pm 0.80$ \\
\hline Globulin & $(\mathrm{g} / \mathrm{L})$ & $32.7 \pm 0.72$ & $33.1 \pm 0.75$ & $32.1 \pm 0.43$ & $34.2 \pm 0.66$ \\
\hline A/G ratio & $(\mathrm{g} / \mathrm{L})$ & $1.14 \pm 0.03$ & $1.14 \pm 0.03$ & $1.18 \pm 0.03$ & $1.12 \pm 0.02$ \\
\hline
\end{tabular}

Values are expressed as mean $\pm \mathrm{SD}$ ( $n=5$ for each group). G1: No treatment; G2: Paraffin;

G3: M. malabathricum $5000 \mathrm{mg} / \mathrm{kg}$; G4: M. malabathricum $2000 \mathrm{mg} / \mathrm{kg}$

\section{Histopathology examination}

The microscopic structures of the liver, kidneys and skin are shown in Figure 2. There are no unnoticeable differences between the control and treatment groups. Microscopic examination was conducted showing that the organs from extracttreated rats had no any alterations in cell structures or any other undesirable effects when viewed under a light microscope using multiple magnification powers. 
Int. J. Curr. Res. Med. Sci. (2017). 3(5): 84-99

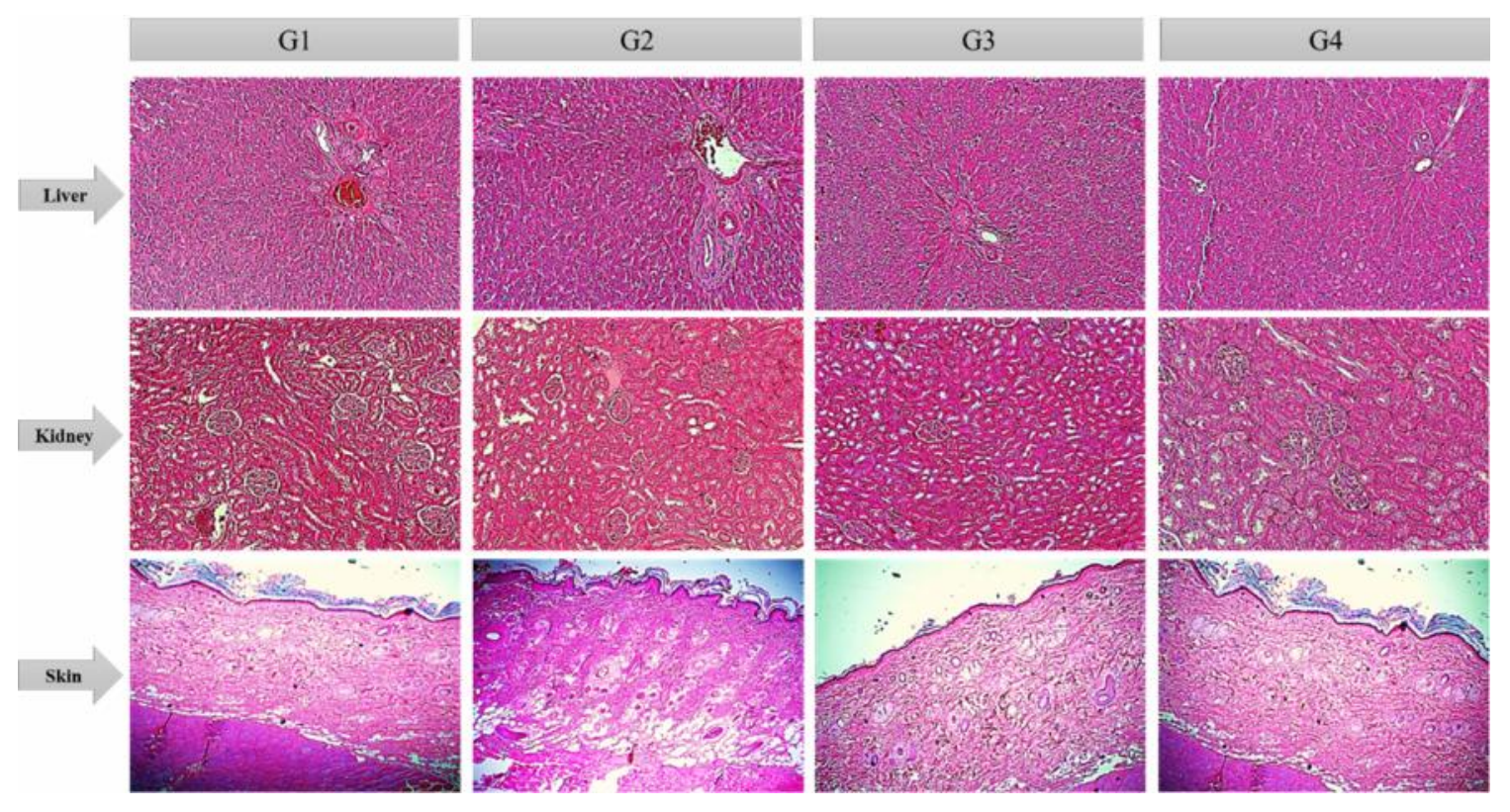

Fig. 2: Histological sections of liver, kidney and skin of rats in all groups. G1: No treatment; G2: Paraffin; G3: M. malabathricum 2000 mg/kg; G4: M. malabathricum 5000 mg/kg. Selected photomicrographs $\times 10$ and $\times 20$. Hematoxylin-eosin staining (scale bar: $200 \mathrm{~m}$ )

\section{Sub-acute dermal toxicity}

\section{General sign and behavioural analysis}

Tables 9 and 10 depict both the toxic effects of ethanolic extracts of $M$. malabathricum in terms of appearance and general behavioural patterns of the examined rats. Toxic signs and/or mortality were not observed in any animals surviving up to 28 days after application of daily different doses of 2000,1000 , and $500 \mathrm{mg} / \mathrm{kg}$ body weight. Behavioural patterns were observed 6 hours and 14 hours after extract application. Both vehicle treated and extract-treated animals appeared normal. None showed any significant changes in skin effects, breathing patterns, postural abnormalities, impairment in food or water consumption, or hair loss. The treated groups had a rapid heartbeat for up to 6 hours after treatment. This is likely due to handling stress.

Table 9: Mortality rate of rats after applied with topical ethanolic extracts of $M$. malabathricum at $2000 \mathrm{mg} / \mathrm{kg}, 1000 \mathrm{mg} / \mathrm{kg}$ and $500 \mathrm{mg} / \mathrm{kg}$, daily, for 28 days

\begin{tabular}{|c|c|}
\hline Group & Mortality rate*(\%) \\
\hline G1 & 0 \\
\hline G2 & 0 \\
\hline G3 & 0 \\
\hline G4 & 0 \\
\hline G5 & 0 \\
\hline
\end{tabular}

* Mortality rate is number of dead rats divided by total number of rats per group.G1: No treatment; G2: Paraffin; G3: M. malabathricum 2000 mg/kg; G4: M. malabathricum 1000 mg/kg; G5: M. malabathricum $500 \mathrm{mg} / \mathrm{kg}$ 
Int. J. Curr. Res. Med. Sci. (2017). 3(5): 84-99

Table 10: Behavioural patterns and general appearance of rats in all groups

\begin{tabular}{|c|c|c|c|c|}
\hline Abnormal sign & $\begin{array}{c}\text { Control group: } \\
\text { G1 }(6 \mathrm{~h})\end{array}$ & $\begin{array}{c}\text { Control } \\
\text { group: } \\
\text { G1 }(14 \mathrm{~h})\end{array}$ & $\begin{array}{c}\text { Treatment } \\
\text { groups: } \\
\text { G2-G5 }(6 \mathrm{~h})\end{array}$ & $\begin{array}{c}\text { Treatment } \\
\text { groups: } \\
\text { G2-G5 }(14 \mathrm{~h})\end{array}$ \\
\hline Skin and fur & Normal & Normal & Normal & Normal \\
\hline Eyes & Normal & Normal & Normal & Normal \\
\hline Mucous membrane & Normal & Normal & Normal & Normal \\
\hline Behavioural patterns & Normal & Normal & Tachycardia & Normal \\
\hline Salivation & Normal & Normal & Normal & Normal \\
\hline Lethargy & Normal & Normal & Normal & Normal \\
\hline Sleep & Normal & Normal & Normal & Normal \\
\hline Diarrhea & Normal & Normal & Normal & Normal \\
\hline Coma & NO & NO & NO & NO \\
\hline Tremors & NO & NO & NO & NO \\
\hline
\end{tabular}

G1: No treatment, G2: Paraffin, G3: M. malabathricum 2000 mg/kg, G4: M. malabathricum 1000 mg/kg, G5: M. malabathricum $500 \mathrm{mg} / \mathrm{kg}$, NO: Not observed

\section{Organ and body weight}

Body weights and weights of liver and kidneys of the rats are shown in Figure 3 and Table 11. No significant changes in body weight were noted. All rats demonstrated a normal increment in the body weight which was not significantly ( $\mathrm{p}>$ 0.05) different between the control and treated groups. Similarly, there were no significant $(\mathrm{p}>$ $0.05)$ differences in changes of relative organ weights between groups.

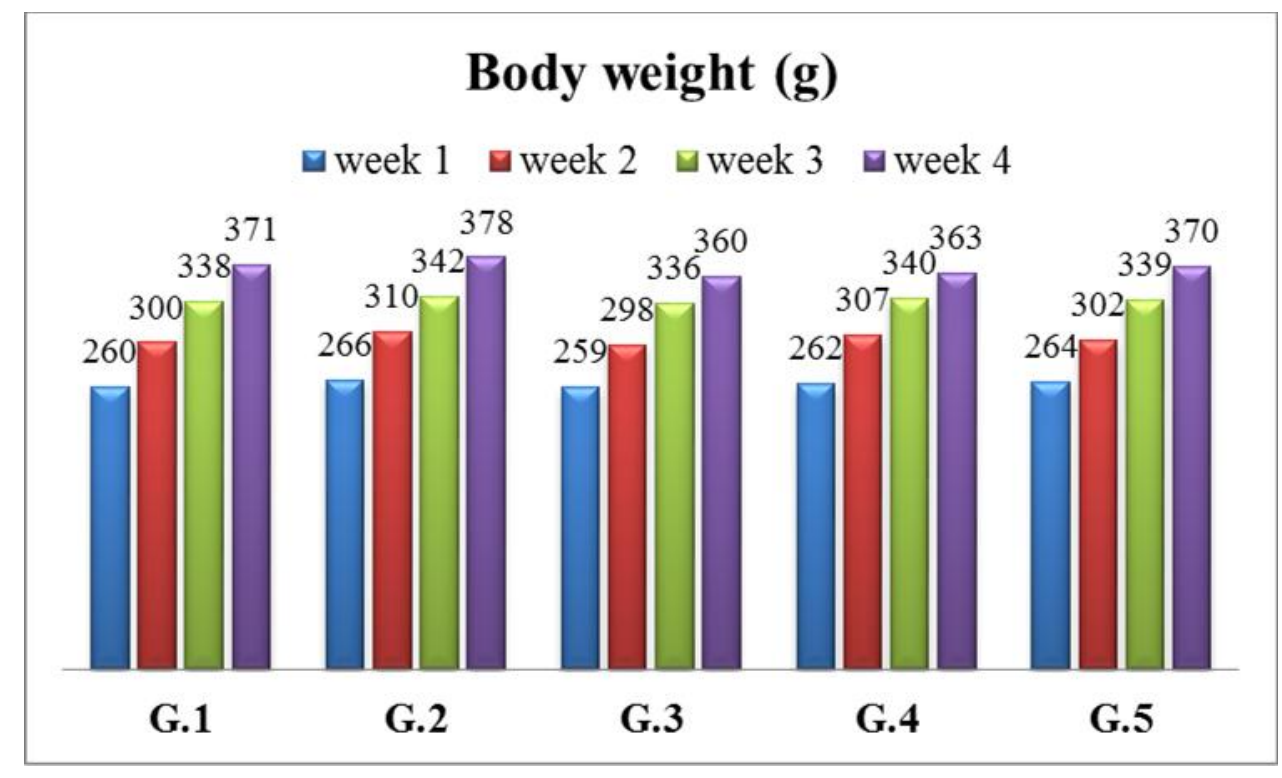

Fig. 3: Mean body weight (g) of rats in all groups. Data collected were recorded and presented as (mean \pm SEM). G1: No treatment group; G2: Paraffin group; G3: M. malabathricum $(2000 \mathrm{mg} / \mathrm{kg})$;

G4: M. malabathricum (1000 mg/kg); G5: M. malabathricum $(500 \mathrm{mg} / \mathrm{kg}$ 
Int. J. Curr. Res. Med. Sci. (2017). 3(5): 84-99

Table 11: Organ relative weight of rats in all groups

\begin{tabular}{|c|c|c|}
\hline Group & Liver $(\mathrm{g})$ & Kidneys $(\mathrm{g})$ \\
\hline G1 & $0.026 \pm 0.002$ & $0.0062 \pm 0.0002$ \\
\hline G2 & $0.031 \pm 0.002$ & $0.0062 \pm 0.0002$ \\
\hline G3 & $0.030 \pm 0.003$ & $0.0064 \pm 0.0002$ \\
\hline G4 & $0.028 \pm 0.005$ & $0.0062 \pm 0.0002$ \\
\hline G5 & $0.029 \pm 0.004$ & $0.0063 \pm 0.0003$ \\
\hline
\end{tabular}

Values are expressed as mean $\pm \mathrm{SD}$ ( $n=6$ for each group). Relative organ weight was calculated by organ weight/body weight $\times 100 \%$. G1: No treatment group; G2: Paraffin group; G3: M. malabathricum (2000 $\mathrm{mg} / \mathrm{kg}) ; \mathrm{G} 4:$ M. malabathricum $(1000 \mathrm{mg} / \mathrm{kg}) ; \mathrm{G} 5:$ M. malabathricum $(500 \mathrm{mg} / \mathrm{kg})$

\section{Haematology evaluation}

In all rats, erythron parameters were normal, as shown in Table 12. Normal results were also observed for the leukon parameters, as shown in Table 13.

Table 12: Erythron parameters and plasma protein concentration of rats in all groups

\begin{tabular}{|c|c|c|c|c|c|c|}
\hline Parameter & Unit & $\begin{array}{c}\text { G1 } \\
\text { (Mean } \\
\pm \text { SD) }\end{array}$ & $\begin{array}{c}\text { G2 } \\
\text { (Mean } \\
\pm \text { SD) }\end{array}$ & $\begin{array}{c}\text { G3 } \\
\text { (Mean } \\
\pm \text { SD) }\end{array}$ & $\begin{array}{c}\text { G4 } \\
\text { (Mean } \\
\pm \text { SD) }\end{array}$ & $\begin{array}{c}\text { G5 } \\
\text { (Mean } \\
\pm \text { SD) }\end{array}$ \\
\hline $\mathrm{RBC}$ & $\mathrm{x} 1012 / \mathrm{L}$ & $8.60 \pm 0.44$ & $8.93 \pm 0.56$ & $8.62 \pm 0.58$ & $8.11 \pm 0.65$ & $8.75 \pm 0.83$ \\
\hline $\mathrm{Hb}$ & $\mathrm{g} / \mathrm{L}$ & $170 \pm 8.57$ & $172 \pm 3.83$ & $176 \pm 9.66$ & $171 \pm 15.9$ & $176 \pm 15.7$ \\
\hline $\mathrm{PCV}$ & $\mathrm{L} / \mathrm{L}$ & $0.45 \pm 0.00$ & $0.46 \pm 0.01$ & $0.44 \pm 0.03$ & $0.45 \pm 0.02$ & $0.43 \pm 0.02$ \\
\hline $\mathrm{MCV}$ & $\mathrm{g} /$ & $57.6 \pm 1.75$ & $57.8 \pm 0.75$ & $58.6 \pm 1.03$ & $57.6 \pm 2.16$ & $57.6 \pm 1.03$ \\
\hline $\mathrm{MCHC}$ & $\mathrm{g} / \mathrm{L}$ & $342 \pm 7.31$ & $345 \pm 7.74$ & $342 \pm 7.08$ & $347 \pm 5.56$ & $348 \pm 5.51$ \\
\hline $\mathrm{PP}$ & $\mathrm{g} / \mathrm{L}$ & $77.6 \pm 1.86$ & $75.3 \pm 1.03$ & $77.3 \pm 1.03$ & $75.5 \pm 1.64$ & $77.3 \pm 1.96$ \\
\hline
\end{tabular}

Values are expressed as mean $\pm \mathrm{SD}$ ( $n=6$ for each group). G1: No treatment; G2: Paraffin; G3: M. malabathricum $2000 \mathrm{mg} / \mathrm{kg}$; G4: M. malabathricum $1000 \mathrm{mg} / \mathrm{kg}$; G5: M. malabathricum 500 $\mathrm{mg} / \mathrm{kg}$.None of the values were significantly different at $\mathrm{P}>0.05$

Table 13: Leukon and thrombon parameters of rats in all groups

\begin{tabular}{|c|c|c|c|c|c|c|}
\hline Parameter & Unit & $\begin{array}{c}\text { G1 } \\
\text { (Mean } \\
\pm \text { SD) }\end{array}$ & $\begin{array}{c}\text { G2 } \\
\text { (Mean } \\
\pm \text { SD) }\end{array}$ & $\begin{array}{c}\text { G3 } \\
\text { (Mean } \\
\pm \text { SD) }\end{array}$ & $\begin{array}{c}\text { G4 } \\
\text { (Mean } \\
\pm \text { SD) }\end{array}$ & $\begin{array}{c}\text { G5 } \\
\text { (Mean } \\
\pm \text { SD) }\end{array}$ \\
\hline WBC & $10^{9} / \mathrm{L}$ & $7.45 \pm 1.31$ & $8.01 \pm 0.74$ & $7.25 \pm 0.87$ & $7.75 \pm 1.33$ & $7.68 \pm 1.22$ \\
\hline Neutrophils & $10^{9} / \mathrm{L}$ & $1.49 \pm 0.98$ & $1.61 \pm 0.75$ & $1.45 \pm 0.89$ & $1.51 \pm 1.36$ & $1.48 \pm 0.81$ \\
\hline Lymphocytes & $10^{9} / \mathrm{L}$ & $5.43 \pm 1.54$ & $5.81 \pm 1.36$ & $5.31 \pm 1.03$ & $5.71 \pm 1.60$ & $5.66 \pm 1.32$ \\
\hline Monocytes & $10^{9} / \mathrm{L}$ & $0.29 \pm 1.09$ & $0.29 \pm 1.21$ & $0.24 \pm 0.51$ & $0.28 \pm 1.21$ & $0.30 \pm 1.26$ \\
\hline Esoinophils & $10^{9} / \mathrm{L}$ & $0.17 \pm 0.81$ & $0.17 \pm 0.75$ & $0.14 \pm 1.09$ & $0.16 \pm 0.75$ & $0.14 \pm 1.16$ \\
\hline Basophils & $10^{9} / \mathrm{L}$ & $0.04 \pm 0.81$ & $0.10 \pm 0.81$ & $0.08 \pm 0.75$ & $0.07 \pm 0.81$ & $0.06 \pm 0.75$ \\
\hline Platelets & $10^{9} / \mathrm{L}$ & $864 \pm 44.9$ & $837 \pm 27.3$ & $785 \pm 69.5$ & $827 \pm 34.7$ & $803 \pm 38.2$ \\
\hline
\end{tabular}

Values are expressed as mean \pm SD ( $n=6$ for each group). G1: No treatment; G2: Paraffin; G3: M. malabathricum 2000 mg/kg; G4: M. malabathricum 1000 mg/kg; G5: M. malabathricum 500 $\mathrm{mg} / \mathrm{kg}$. None of the values were significantly different at $\mathrm{P}>0.05$ 


\section{Blood biochemistry}

Serum biochemical parameters of liver and muscle enzymes are shown in Table 14. Table 15 contains kidney parameters while protein concentrations are shown in Table 16 for both the male rats of the control and M. malabathricum extract groups. No significant $(\mathrm{p}>0.05)$ changes were observed in any of the biochemical parameters.

Table 14: Serum biochemical parameters of liver and muscle enzymes of rats in all groups

\begin{tabular}{|c|c|c|c|c|c|c|}
\hline Parameter & Unit & $\begin{array}{c}\text { G1 } \\
\text { (Mean } \\
\pm \text { SD) }\end{array}$ & $\begin{array}{c}\text { G2 } \\
(\text { Mean } \\
\pm \text { SD) }\end{array}$ & $\begin{array}{c}\text { G3 } \\
(\text { Mean } \\
\pm \text { SD })\end{array}$ & $\begin{array}{c}\text { G4 } \\
\text { (Mean } \\
\pm \text { SD) }\end{array}$ & $\begin{array}{c}\text { G5 } \\
\text { (Mean } \\
\pm \text { SD) }\end{array}$ \\
\hline ALT & (U/L) & $44.1 \pm 8.35$ & $42.0 \pm 6.41$ & $38.5 \pm 6.34$ & $42.5 \pm 7.06$ & $44.1 \pm 7.35$ \\
\hline ALP & (U/L) & $135 \pm 12.3$ & $138 \pm 10.1$ & $130 \pm 12.6$ & $131 \pm 10.7$ & $125 \pm 13.3$ \\
\hline AST & (U/L) & $149 \pm 12.4$ & $150 \pm 16.5$ & $139 \pm 19.4$ & $123 \pm 18.1$ & $145 \pm 13.5$ \\
\hline CK & (U/L) & $201 \pm 22.1$ & $221 \pm 26.3$ & $202 \pm 14.2$ & $221 \pm 19.5$ & $213 \pm 26.2$ \\
\hline
\end{tabular}

Values are expressed as mean $\pm \mathrm{SD}$ ( $n=6$ for each group). G1: No treatment group; G2: Paraffin group; G3: M. malabathricum (2000 mg/kg); G4: M. malabathricum (1000 mg/kg); G5: M. malabathricum (500 $\mathrm{mg} / \mathrm{kg}$. None of the values were significantly at $\mathrm{P}>0.05$

Table 15: Serum biochemical parameters of kidney of rats in all groups

\begin{tabular}{|c|c|c|c|c|c|c|}
\hline Parameter & Unit & $\begin{array}{c}\text { G1 } \\
\text { (Mean } \\
\pm \text { SD) }\end{array}$ & $\begin{array}{c}\text { G2 } \\
\text { (Mean } \\
\pm \text { SD) }\end{array}$ & $\begin{array}{c}\text { G3 } \\
\text { (Mean } \\
\pm \text { SD) }\end{array}$ & $\begin{array}{c}\text { G4 } \\
\text { (Mean } \\
\pm \text { SD) }\end{array}$ & $\begin{array}{c}\text { G5 } \\
\text { (Mean } \\
\pm \text { SD) }\end{array}$ \\
\hline Urea & $(\mathrm{mmol} / \mathrm{L})$ & $7.71 \pm 0.91$ & $6.91 \pm 0.57$ & $7.50 \pm 0.73$ & $7.18 \pm 0.88$ & $7.08 \pm 0.66$ \\
\hline Creatinine & $(\mathrm{mol} / \mathrm{L})$ & $55.5 \pm 2.25$ & $55.5 \pm 3.08$ & $51.5 \pm 1.22$ & $54.3 \pm 2.42$ & $51.8 \pm 2.63$ \\
\hline
\end{tabular}

Values are expressed as mean $\pm \mathrm{SD}$ ( $n=6$ for each group). G1: No treatment group; G2: Paraffin group; G3: M. malabathricum (2000 mg/kg); G4: M. malabathricum $(1000 \mathrm{mg} / \mathrm{kg}) ; \mathrm{G} 5:$ M. malabathricum (500 $\mathrm{mg} / \mathrm{kg}$. None of the values were significantly different at $\mathrm{P}>0.05$

Table 16: Serum biochemical parameters of protein concentration of rats in all groups

\begin{tabular}{|c|c|c|c|c|c|c|}
\hline Parameter & Unit & $\begin{array}{c}\text { G1 } \\
\text { (Mean } \\
\pm \text { SD })\end{array}$ & $\begin{array}{c}\text { G2 } \\
(\text { Mean } \\
\pm \text { SD })\end{array}$ & $\begin{array}{c}\text { G3 } \\
(\text { Mean } \\
\pm \text { SD })\end{array}$ & $\begin{array}{c}\text { G4 } \\
\text { (Mean } \\
\pm \text { SD })\end{array}$ & $\begin{array}{c}\text { G5 } \\
(\text { Mean } \\
\pm \text { SD })\end{array}$ \\
\hline TP & $(\mathrm{g} / \mathrm{L})$ & $72.2 \pm 4.09$ & $75.5 \pm 4.37$ & $72.2 \pm 2.77$ & $72.2 \pm 5.29$ & $70.4 \pm 2.97$ \\
\hline Albumin & $(\mathrm{g} / \mathrm{L})$ & $40.8 \pm 1.15$ & $43.8 \pm 2.62$ & $41.3 \pm 2.01$ & $39.3 \pm 3.61$ & $38.8 \pm 2.25$ \\
\hline Globulin & $(\mathrm{g} / \mathrm{L})$ & $31.4 \pm 3.23$ & $31.6 \pm 5.26$ & $30.9 \pm 1.84$ & $32.8 \pm 3.06$ & $31.6 \pm 3.02$ \\
\hline A/G ratio & $(\mathrm{g} / \mathrm{L})$ & $1.25 \pm 0.13$ & $1.36 \pm 0.26$ & $1.30 \pm 0.10$ & $1.16 \pm 0.12$ & $1.20 \pm 0.15$ \\
\hline
\end{tabular}

Values are expressed as mean $\pm \mathrm{SD}$ ( $n=6$ for each group). G1: No treatment group; G2: Paraffin group; G3: M. malabathricum (2000 mg/kg); G4: M. malabathricum (1000 mg/kg); G5: M. malabathricum (500 $\mathrm{mg} / \mathrm{kg}$. None of the values were significantly different at $\mathrm{P}>0.05$

\section{Histopathology examination}

The liver, kidneys and skin shown in Figure 4 have no noticeable differences between control and treatment groups. No organs from the extract treated rats demonstrated any alteration in cell structure or other negative effects when examined under a light microscope with various magnification powers. 


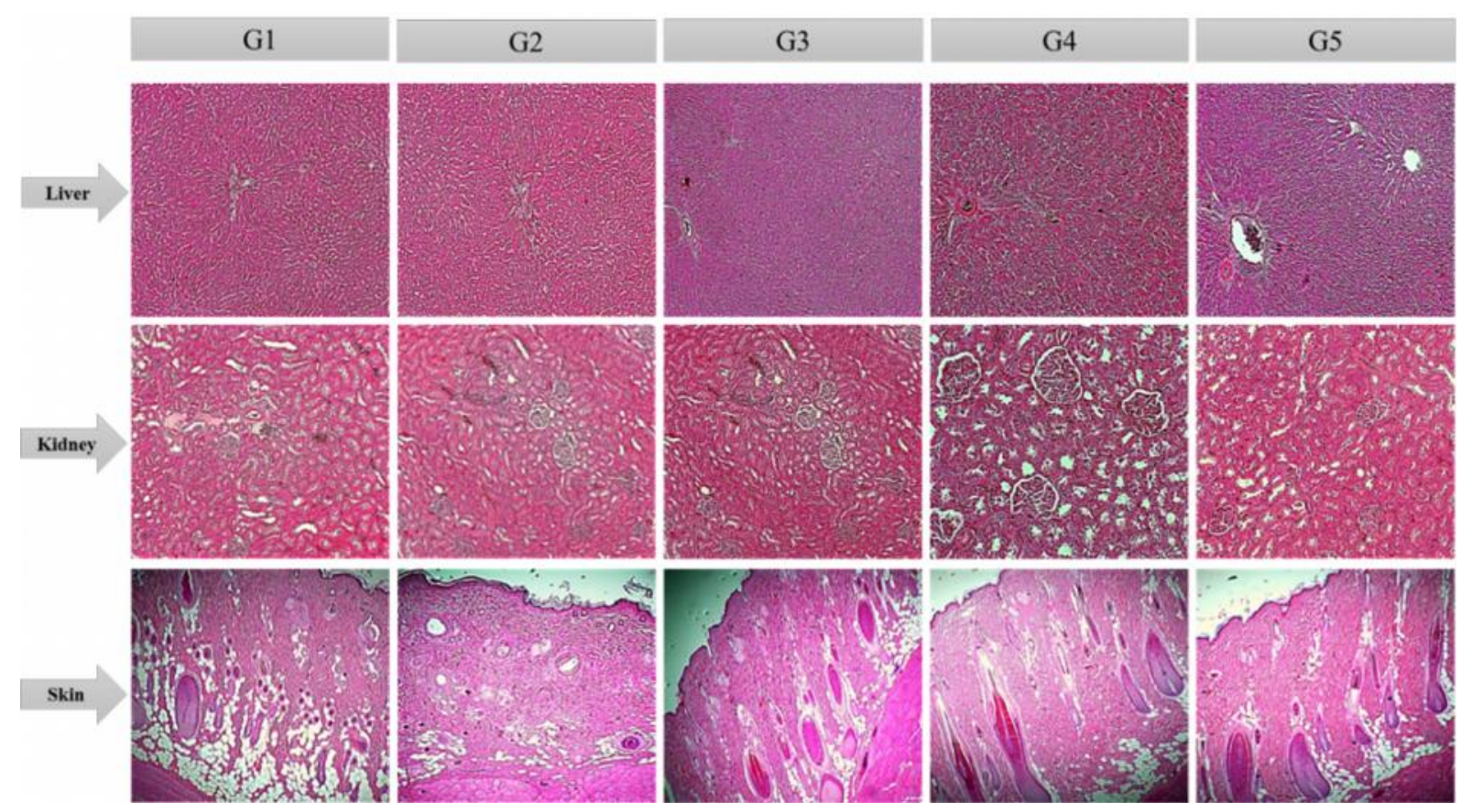

Fig. 4: Histological sections of liver, kidney and skin of rats in all groups G1: No treatment; G2: Paraffin;

G3: M. malabathricum 500 mg/kg; G4: M. malabathricum 1000 mg/kg; G5: M. malabathricum 2000 $\mathrm{mg} / \mathrm{kg}$. Selected photomicrographs $\times 10$ and $\times 20$. Hematoxylin-eosin staining (scale bar: $200 \mathrm{~m}$ )

\section{Discussion}

Phytotherapeutic products from medicinal plants have come to be widely used in primary healthcare in developing countries. Some such products are considered safe due to their natural providence. Bioactive products from medicinal plants tend to be used more often in selfmedication due to being considered naturally safe (Vaghasiya et al., 2011). However, this is a dangerous assumption, as there are many such plant compounds which are highly toxic, including the most cytotoxic anti-cancer plantderived drugs, digitalis, the pyrrolizidine alkaloids, ephedrine, phorbol esters, and so on (Vaghasiya et al., 2011). Most herbal drugs show less harmful side effects when the drugs are appropriately used, when compared with synthetic drugs (Kirtikar and Basu, 1975). Toxic substances may have compelling pharmacological effects at a lower, nontoxic dose. Toxicity results as found through animal testing are important in determining the safety and usefulness of pharmacologically active plants (Moshi and Brine shrimp, 2007). Worldwide, use of such medicinal plants is growing; however, there remains a lack of knowledge about the relative toxicity and potential adverse effects of such plants. Further in vivo studies are called for to determine signs of toxicity as well as the correct range of dosages of such plants (Syahmi et al., 2010).

Acute and sub-acute toxicity testing in laboratory animal are used to evaluate natural remedies for different pharmacological activities (Sasidharan et al., 2008). It is one of the necessary studies need to be performed for the toxicological analyses of medicinal plants. The toxicity effects of the plants are evaluated through qualitative and quantitative analyses of blood and histopathology samples of the laboratory animal (Sasidharan et al., 2008). The present study was designed to investigate the toxicity of ethanolic extracts of M. malabathricum, for topical application via acute and sub-acute dermal toxicity analyses. However, results in this study indicated no signs of toxicity and deaths observed throughout the experimental period in both acute and sub-acute toxicity studies. 
No rats topically exposed to the ethanolic extracts of $M$. malabathricum demonstrated any organ or systemic toxicity at $2000 \mathrm{mg} / \mathrm{kg}$ and $5000 \mathrm{mg} / \mathrm{kg}$ in the acute dermal toxicity study or dosages of $500 \mathrm{mg} / \mathrm{kg}, 1000 \mathrm{mg} / \mathrm{kg}$ and $2000 \mathrm{mg} / \mathrm{kg}$ in subacute dermal toxicity study. Rats were monitored daily for signs of toxicity or mortality until day 14 in the acute study and until day 28 in the subacute study. During this monitoring period, rats showed no overt signs of distress. No symptoms toxicity was noted and there were no deaths. Clinical abnormality is one of the major important observations which indicate toxicity effects on organs in the treated groups (Eaton and Klaassen, 1996). No rats displayed significant changes in behaviour.

In addition, the physical appearances such as skin, fur and eyes were normal and whilst the body weight of the rats was increased, this indicates that applying of the crude extracts on the skin had negligible level of toxicity on the growth of the rats. Moreover, proper food and water intake is essential for a therapeutic product safety study. Proper nutrition has a significant effect on animal status and on the measured effects of the drugs to be tested (Steven and Iversen, 2003). In this study, food and water consumption remained normal. This lack of an effect on appetite means there was no disturbance in carbohydrate, protein or fat metabolism levels (Klaassen, 2001). Typically, body weight and internal organ weight gain changes are a measure of toxicity after toxic substance exposure (Carol, 1995). In 2002, a study by Raza and his team found that body weight changes are indicators of adverse effects of drugs and chemicals. These changes become relatively more significant if body weight loss exceeds $10 \%$. Organ weight also is an important index of physiological and pathological status in animals.

The relative organ weight is fundamental to diagnose whether the organ was exposed to the injury or not (Teoet al., 2002). Liver, kidneys and spleen are the primary organs affected by metabolic reaction caused by toxicant (Dybing et al., 2002). In this study, gross appearance of the selected organs of both control and treated groups were normal. The relative and absolute weight of the organs in both control and treated groups showed no significant differences. Body weight gain was similar in both control and treated groups without statistically significant differences. Local application of ethanolic extracts of $M$. malabathricum was not shown to cause adverse effects on organ weight of any important organs. This suggests that M. malabathricum extracts are virtually nontoxic. The haematopoietic system tends to be quite sensitive to toxic compounds. It acts as an important index of physiological and pathological status in both animals and humans (Adeneye et al., 2006). After 14 and 28 days of treatment with ethanolic extracts of $M$. malabathricum, no changes were found in the haematological parameters of either the control or treatment groups. Also, no significant changes in levels of all serum biochemistry parameters were found.

This evidences the nontoxic nature of ethanolic extract of $M$. malabathricum. This finding is in the agreement with the safe use of the herbs by the traditional healers. One study performed in 1999 by Ajagbonna and his team found that the normal range of haematological parameters may be changed through the ingestion of toxic plants. Histopathology may be used for confirmation of cell structure alteration due to toxicity. Histopathological examination remains the gold standard method for evaluating treatment-related pathological changes in tissues and organs (OECD, 1995). The histopathological evaluation conducted for this study to determine acute and sub-acute dermal toxicity has indicated that ethanolic extracts of $M$. malabathricum did not cause any structural damage in the morphology of liver, kidneys and skin.

Histopathological findings matched with the results of haematological and biochemical analyses. The histopathology results were crosscorrelated with body weight and relative organ weights. In this study, the liver histology revealed normal hepatocytes; portal and central vein, bile duct as well as hepatic artery did not show any alterations in the structure in both controls and treated rats. In contrast to the present study, the histological examination of a study conducted by Harizal and his team in 2010 using Mitragyna speciosa extract revealed morphological changes 
in liver of mice treated with the extract at dose levels of $100 \mathrm{mg} / \mathrm{kg}$ and $500 \mathrm{mg} / \mathrm{kg}$. Meanwhile, in 2009 another study by Salawuusing Crossopteryx febrifuga observed inflammatory changes histologically in the liver by infiltration of lymphocytes at portal and central veins of rats treated at dose levels of $500 \mathrm{mg} / \mathrm{kg}$ and 1000 $\mathrm{mg} / \mathrm{kg}$. Those studies showed that the extracts exerted deleterious effects on the liver.

Liver is capable of regenerating damaged tissues, hence it functions may not be impaired early on following an insult from a toxicant (Salawu et al., 2009). Additionally, one toxicity study conducted on a Cassia fistula pod extract performed by (Akanmu et al., 2004) reported that the histological examinations of liver, kidneys, and testes showed. No potential toxicity or damage to the cell structure of the liver, kidneys and testes at a dose of $1000 \mathrm{mg} / \mathrm{kg}$ extract were found. No necrosis, inflammatory reaction, fibrosis, or local fatty degeneration were observed in the liver or hepatocytes. The morphology of liver cells in both the control and treated groups of this were also normal. No structural alterations were observed under the microscope. As with the liver, kidney microphotograph histology found no adverse effects in either group. A previous study by (Alade et al., 2009) found focal proximal tubular epithelial necrosis in the kidneys. Another study by (Akanmu et al., 2004) on the effects of C. fistula pod extract in rats revealed were significant changes in the histology of kidneys treated with the extract at a dose of $1000 \mathrm{mg} / \mathrm{kg}$.

In this present study, the microscopic examination of the skin of rats treated with the extracts did not indicate any changes in the layers of the skin at the epidermis, dermis and hypodermis as compared to the control rats. Both glomeruli and Bowman's capsules were normal. Colloidal Nano silver toxicity at doses of 100, 1000 and 10000 $\mathrm{g} / \mathrm{mL}$ in guinea pigs was shown to cause skin lesions. This indicates a decreased thickness of epidermis and dermis, increased numbers of Langerhans and inflammatory cells, decreased papillary layer with regular collagens fibres, acidophilic cytoplasm in muscle fibres, degenerative fibres, and increased levels of macrophage in the endomysium (Korani et al., 2011).

In conclusion, study results suggest that ethanolic extracts of $M$. malabathricum cause no obvious in vivo toxicity. Deathor signs of acute toxicity were not observed in rats treated with doses of 5000and $2000 \mathrm{mg} / \mathrm{kg}$ (acute toxicity study). The same holds for those rats treated with doses of 2000 $\mathrm{mg} / \mathrm{kg}, 1000 \mathrm{mg} / \mathrm{kg}$, and $500 \mathrm{mg} / \mathrm{kg}$ (sub-acute toxicity study). This indicates that the extract is safe to use. Histology examinations also found no change in the architectures of selected organs for both the control and treated groups. According to the findings of this research, $M$. malabathricum is suitable for use as a topical medicinal agent at those dosages. This is especially indicated in rural communities, for which conventional drugs are unaffordable.

\section{References}

Adeneye, A.A., Ajagbonna, O.P., Adeleke, T.I., Bello, S.O. 2006. Preliminarytoxicity and phytochemical studies of the stem bark aqueous extract of Musanga cecropioides in rats. J. Ethnopharmacol 105, 374-379.

Ajagbonna, O.P., Onifade, K.I., Suleiman, U. 1999. Haematological and biochemical changes in rats given extract of $C$. pocera. Sokoto. J. Vet. Sci. 1, 3-12.

Akanmu, M.A., Iwalewa, E.O., Elujoba, A.A., Adelusola, K.A. 2004. Toxicity potentials of Cassia fistula fruits as laxative with reference to senna. Afr. J.Biomed. Res. 7, 23-26.

Alade, G.O., Akanmu, M.A., Obuotor, E.M., Osasan, S.A., Omobuwajo, O.R. 2009. Acute and oral sub-acute toxicity of methanolic extract of Bauhinia monandra leaf in rats. Afr. J. Pharm. Pharmacol3, 354-358.

Asante-Duah, K. 2002. Public Health Risk Assessment for Human Exposure to Chemicals (illustrated.); Kluwer Academic Publishers: Dordrecht, The Netherlands. Volume 6.

Carol, S.A. 1995. Acute, Sub-chronic and Chronic Toxicology. In CRC Handbook of Toxicology; Michael, J.D., Mannfred, A.H., Eds. CRC Press Inc. Boca Raton, FL, USA 51-104. 
Dybing, E., Doe, J., Groten, J., Kleiner, J., O'Brien, J. 2002. Hazard characterization of chemicals in food and diet: dose response, mechanism and extrapolation issues. Food Chem. Toxicol 42, 237-282.

Eaton, D.L., Klaassen, C.D. 1996. Principles of toxicology. In Casarett and Doull's Toxicology: The Basic Science of Poisons, 5th ed; Klaassen, C.D. Ed.; McGraw-Hill: New York, NY, USA p. 13.

Grosvenor, P.W., Gothard, P.K., McWilliam, N.C., Supriono, A. and Gray, D.O. 1995. "Medicinal plants from Riau Province, Sumatra, Indonesia. Part 1: uses, "Journal of Ethnopharmacology, vol. 45, no. 2, pp. 75-95.

Harizal, S.N., Mansor, S.M., Hasnan, J., Tharakan, J.K.J., Abdullah, J. 2010. Acute toxicity study of the standardized methanolic extract of Mitragyna speciosa Korthin Rodent. J. Ethnopharmacol 131, 404-409.

Iversen, P.O., Nicolaysen, G. 2003. Water for life. J. Norw. Med. Assoc 123, 3402-3405.

Kirtikar, K.R., Basu, B.D. 1975. Indian Medicinal Plants; International Book Distributors: Dehradun, India, Volume 2, p. 858.

Klaassen, C.D. 2001. Principles of Toxicology. In Casarett and Doull's Toxicology: The Basic Science of Poisons, 5th ed.; McGraw-Hill: New York, NY, USA, p. 13.

Koay, S.S. 2008. Establishment of cell suspension culture of Melastoma malabathricum L. for the production of anthocyanin, Ph.D. thesis, UniversitiSains Malaysia, Pulau Pinang, Malaysia.

Korani, M., Rezayat, S.M., Gilani, K.,ArbabiBidgoli, S., Adeli, S. 2011. Acute and subchronic dermal toxicity of nanosilver in guinea pig. International Journal ofNanomedicine6: 855-862.

Lipnick, R.L., Cotruvo, J.A., Hill, R.N., Bruce, R.D., Stitzel, K.A., Walker, A.P.,Chu, I., Goddard, M., Segal, L., Springer, J.A. and Myers, R.C. 1995. Comparison of the up-and down, conventional LD50, and fixed-dose acute toxicity procedures. Fd. Chem. Toxicol. 33: 223-231.
Meyer, K. 2001. "Revision of the Southeast Asian genus Melastoma (Melastomataceae)," Blumea: Journal of Plant Taxonomy and Plant Geography, vol. 46, no. 2, pp. 351-398.

Moshi, M.J. 2007. Brine shrimp toxicity evaluation of some Tanzanian plants used traditionally for the treatment of fungal infections. Afr. J. Tradit. Complement. Altern. Med 4, 219-225.

OECD. 1995. OECD Guideline for Testing of Chemicals. Repeated Dose 28-DayOral Toxicity Study in Rodents; Organisation for Economic Co-operation and Development: Paris, France.

Rajenderan, M.T. 2010. "Ethno medicinal uses and antimicrobial properties of Melastoma malabathricum," SEGi Review, vol. 3, pp. 3444.

Raza, M., Al-Shabanah, O.A., El-Hadiyah, T.M., Al-Majed, A.A. 2002. Effect of prolonged vigabatrin treatment on haematological and biochemical parameters in plasma, liver and kidney of Swiss albino mice. Sci. Pharm 70, 135-145.

Salawu, O.A., Chindo, B.A., Tijani, A.Y., Obidike, I.C., Salawu, T.A., James Akingbasote, A. 2009. Acute and sub-acute toxicological evaluation of the methanolic stem bark extract of Crossopteryx febrifuga in rats. Afr. J. Pharm. Pharmacol 3, 621-626.

Sasidharan, S., Darah, I., Jain, K. 2008. In vivo and in vitro toxicity study of Gracilaria changii. Pharm.Biol 46, 413-417.

Sunilson, J.A.J., James, J.J., Thomas, J., Jayaraj, P., Varatharajan, R. and Muthappan, M. 2008. "Antibacterial and wound healing activities of Melastoma malabathricum Linn.," African Journal of Infectious Disease, vol. 2, pp. 68-73. Syahmi, A.R.M., Vijayarathna, S., Sasidharan, S., Yoga Latha, L., Kwan, Y.P., Lau, Y.L., Shin, L.N., Chen, Y. 2010. Acute oral toxicity and brine shrimp lethality of Elaeis guineensis Jacq., (Oil Palm leaf) methanol extract. Molecules 15, 8111-8121.

Teo, S.D., Stirling, S., Thomas, A., Kiorpes, A., Vikram, K. 2002. A 90-day oral gavage toxicity study of D-methylphenidate and D, L methylphenidate in Sprague-dawley rats. Toxicology 179,183-196. 
Vaghasiya, Y.K., Shukla, V.J., Chanda, S.V. 2011. Acute oral toxicity study of Pluchea argutaboiss extract in mice. J. Pharmacol. Toxicol 6, 113-123.

Zakaria, M. and Mohd, M.A. 1994. Traditional Malay Medicinal Plants, Fajar BaktiSdn. Bhd., Kuala Lumpur, Malaysia.

\begin{tabular}{|c|l|}
\hline \multicolumn{2}{|c|}{ Access this Article in Online } \\
\hline Q & Website: \\
& www.ijcrims.com \\
\cline { 1 - 1 } & Subject: \\
Quick Response Code & \\
\hline
\end{tabular}

How to cite this article:

Ali Khairullah Zahi, Hazilawati Hamzah, Mohd Rosly Shaari, Riyanto Teguh Widodo, Lucy Johnny, Noordin M.M, and Shanmugavelu Sithambaram. (2017). Investigation and evaluation of acute and subacute dermal toxicity studies of ethanolic leaves extract of Melastoma malabathricum in Sprague Dawley rats. Int. J. Curr. Res. Med. Sci. 3(5): 84-99.

DOI: http://dx.doi.org/10.22192/ijcrms.2017.03.05.013 